\title{
First vortex entry into a perpendicularly magnetized superconducting thin film
}

\author{
J. Gutierrez, ${ }^{1, *}$ B. Raes, ${ }^{1,}{ }^{*}$ J. Van de Vondel, ${ }^{1}$ A. V. Silhanek, ${ }^{1,2}$ R. B. G. Kramer, ${ }^{1,3}$ G. W. Ataklti, ${ }^{1}$ and V. V. Moshchalkov ${ }^{1}$ \\ ${ }^{1}$ INPAC - Institute for Nanoscale Physics and Chemistry, K.U.Leuven, Celestijnenlaan 200D, B-3001 Leuven, Belgium \\ ${ }^{2}$ Département de Physique, Université de Liège, Bât. B5, Allée du 6 août, 17, B-4000 Sart Tilman, Belgium \\ ${ }^{3}$ Institut Néel, CNRS, Université Joseph Fourier, BP 166, 38042 Grenoble Cedex 9, France
}

(Received 15 October 2013; published 8 November 2013)

\begin{abstract}
In type-II superconductors, the flux-free state (Meissner state) may be invaded by vortices bearing quantized flux once $H$ is above the lower critical field $H_{c 1}$. However, the actual first flux penetration does not occur at $H_{c 1}$ due to the presence of a surface barrier and the fact that the Meissner state may also exist as a metastable state up to a larger (superheating) field. In this work we determine the field for the first vortex penetration in superconductors by directly imaging the first vortex threading a superconducting $\mathrm{Pb}$ film with antidots. We find that the first vortex penetration occurs when the surface superconducting currents reach the depairing current, locally breaking superconductivity and allowing a vortex to nucleate.
\end{abstract}

DOI: 10.1103/PhysRevB.88.184504

PACS number(s): 74.78.-w, 74.20.-z, 74.25.Op, 74.81.Fa

\section{INTRODUCTION}

At which field do vortices first appear in a zero-field-cooled type-II superconductor? Vortex entry is thermodynamically allowed as soon as the applied field $H$ becomes larger than the critical field $H_{c 1}$. ${ }^{1}$ This critical field is defined as the field above which the Gibbs free energy of a vortex in a superconducting volume becomes negative.

However, the Meissner state can remain thermodynamically metastable $\mathrm{e}^{2-5}$ at higher magnetic fields, $H>H_{c 1}$, up to the so-called superheating field $H_{\text {sh }}$ at which the first vortex penetrates. The determination of the field $H_{\text {sh }}$ and the underlying criteria for first vortex entry has a long history. The pioneering work $^{2}$ of Bean and Livingston (BL) describes, within the London limit, the effect of a surface on vortex penetration. They considered a vortex inside a type-II superconducting half space parallel to a smooth surface. Close to the surface, the vortex' supercurrent distribution is distorted, as described by an image antivortex. This results in an attractive force between the vortex and the surface. On the other hand, the vortex experiences a repulsive Lorentz force at the surface arising from the screening currents induced by the external magnetic field. The competition between both results in the so-called BL surface barrier for vortex entry. For fields smaller than the BL surface-barrier field $H_{\mathrm{BL}}$ a vortex parallel to the surface cannot penetrate, even though a vortex solution is thermodynamically allowed in the bulk for lower fields. It was shown in Ref. 2 that

$$
H_{\mathrm{BL}}=\frac{H_{c}}{\sqrt{2}}=\frac{\Phi_{0}}{4 \pi \mu_{0} \lambda(T) \xi(T)},
$$

where $H_{c}$ is the thermodynamical critical field, defined as the condensation energy at zero external field, and $\lambda(T)$ and $\xi(T)$ are the temperature-dependent Ginzburg-Landau penetration depth and coherence length, respectively.

Furthermore, $H_{\mathrm{BL}}$ does not yet represent the field determining first flux penetration since it ignores the nucleation of the vortex at the border. ${ }^{6}$ It was shown in Refs. 3-5,7, and 8, by investigating the onset of instability of the Meissner solution using the Ginzburg-Landau formalism, that the unavoidable process of vortex entry into a superconductor is fulfilled when the Meissner screening-current density at an ideal defect-free surface approaches the depairing current density $j_{d}$ :

$$
j_{\text {edge }}=j_{d}=\frac{H_{c}}{\left[\frac{3 \sqrt{6}}{4} \lambda(T)\right]} .
$$

The difference between Eq. (2) and the London expression for the depairing current ${ }^{1}$ is given by the factor $3 \sqrt{6} / 4=$ 1.84. This factor results from the decrease of the density of states with increasing current and is described within the first Ginzburg-Landau equation, which expresses conservation of energy and effectively couples the Cooper pair density with the pair velocity.

The sample geometry can play a determining role in the vortex penetration, as it influences the current distribution within and at the edges of the sample. For example, in thin films which have a large demagnetization factor in a perpendicular field, the strong curvature of the field lines at the edges results in a strongly modified current distribution. When the sample is thicker than the penetration depth, a competition between the line tension of a vortex cutting through the upper and lower ridges at the sample edge and the Lorentz force induced by the Meissner currents results in a so-called geometrical barrier. ${ }^{9,10}$ When the sample thickness is smaller than the penetration depth, vortices are unable to tilt. However, the distortion of the supercurrent flow caused by the strip geometry has profound effects for the first vortex nucleation, resulting in a so-called edge barrier. ${ }^{11}$

Indirect experimental verification of the superheating field and the correlation with the above-described models was based on detecting the onset of the nonlinearity of the initial magnetization in bulk superconductors. ${ }^{12-14}$ We propose here a more direct and reliable way to determine the superheating field by imaging, with single-vortex resolution, the first entered vortex in a thin superconductor. In order to achieve this goal, a careful sample design and fabrication are necessary. First, the presence of surface defects may create spots for premature entry of vortices, ${ }^{4,15,16}$ thus influencing the study of the intrinsic mechanisms. Second, in thin-film geometry, vortices will move to the center of the sample as soon as they nucleate, ${ }^{17}$ making it impossible to experimentally observe their entry. The former problem can be solved by using a superconductor with well-defined edges, whereas creating an 


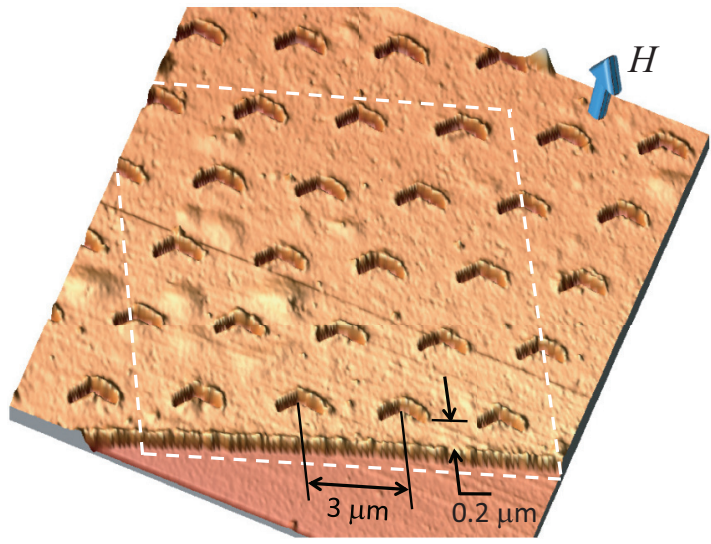

FIG. 1. (Color online) Atomic force microscopy image of sample A. The scanning Hall microscopy images of this work have been taken in a region of the sample with the same size (12- $\mu \mathrm{m}$ side) and same antidot distribution as the one delimited by the dashed lines. Notice that the first row of boomerang-shaped antidots is only $0.2 \mu \mathrm{m}$ away from the edge of the film. The arrow indicates the direction of the applied field perpendicular to the plane of the film.

array of antidots acting as pinning centers allows one to keep the entered vortices relatively close to the sample's edge, where they can be visualized.

\section{SAMPLE DETAILS}

In this work we study two high-quality $\mathrm{Pb}$ superconducting strips with widths of $2 a=300 \mu \mathrm{m}$ (sample A) and $2 a=$ $600 \mu \mathrm{m}$ (sample B) and thicknesses $t=50 \mathrm{~nm}$. Sample A contains a triangular array of boomerang-shaped antidots with an antidot void area of $0.28 \mu \mathrm{m}^{2}$, and sample B contains a square array of square antidots with an antidot void area of $0.36 \mu \mathrm{m}^{2}$. The periodicity of both antidot lattices is $3 \mu \mathrm{m}$. Figure 1 shows a representative atomic force microscopy image of sample A's surface. Notice that the antidots are slightly separated from the border in order to avoid spots of premature flux entry. Further details about sample preparation can be found in Ref. 18. In all cases the magnetic field is applied perpendicularly to the plane of the film. Local magnetic field measurements were carried out via scanning Hall probe microscopy (SHPM) near the sample's border. It is important to remark that although previous works have shown spatially resolved flux penetration in bulk and thin films, ${ }^{19}$ the power of SHPM allows us to determine unambiguously the flux penetration with unprecedented resolution of a single vortex.

In samples with periodic arrays of antidots, stable vortex arrangements are expected for every value of the field at which the vortex lattice is commensurate with the antidot lattice, as theoretically studied via molecular dynamics simulations in Refs. 20 and 21. A way to quantify the quality of the samples consists of imaging at low matching fields. In this case the vortex-vortex interaction is weak, and therefore, small imperfections on the pattern have a sizable impact on the vortex distribution. Experimentally, we can obtain near-toequilibrium vortex distributions by performing field-cooling experiments. Some examples of the resulting flux patterns for sample A directly visualized via SHPM at $T=6.9 \mathrm{~K}$ (a) $H=0.33 H_{1}$

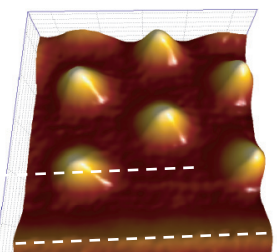

(b) $H=0.52 H_{1}$

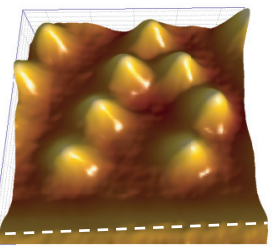

(c) $H=0.63 H_{1}$

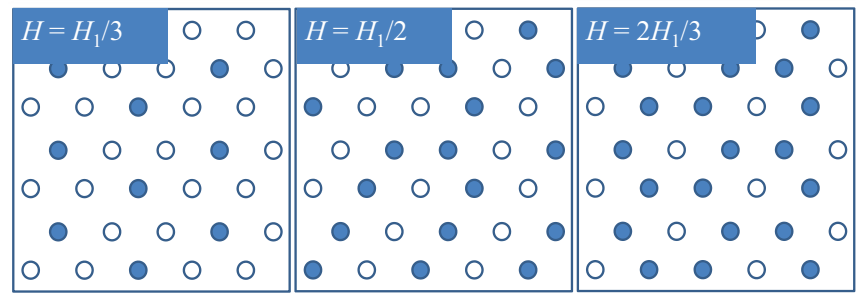

FIG. 2. (Color online) (top) Scanning Hall probe microscopy images obtained for sample A at the sample's edge, as indicated by the dashed box in Fig. 1, after cooling down to $T=6.9 \mathrm{~K}$ in the presence of a field (a) $H=0.33 H_{1}$, (b) $H=0.52 H_{1}$, (c) and $H=0.63 H_{1}$. The dashed line shows the sample's edge. (bottom) Schematic representation of the expected vortex patterns according to Ref. 21. Here the open (solid) circles represent empty (occupied) pinning sites.

and for different applied fields are shown in the top row of Fig. 2. The observed submatching vortex patterns at $H=$ $0.33 H_{1}$ [Fig. 2(a)], $H=0.52 H_{1}$ [Fig. 2(b)], and $H=0.63 H_{1}$ [Fig. 2(c)], where $H_{1}$ is the first matching field, are in agreement with previous theoretical predictions ${ }^{21}$ schematically depicted in the bottom row of Fig. 2. It is important to emphasize that the mere existence of regular patterns in the above field-cooling (FC) experiments is indicative of a low dispersion in pinning energy among different antidots and the fingerprint of a highly homogeneous sample. ${ }^{22-24}$

\section{EXPERIMENTAL RESULTS}

We now focus on the vortex entry under the zero-fieldcooling condition. Figures 3(b)-3(d) show SHPM images at the same spot as in Fig. 2, next to the sample's edge, at $T=7 \mathrm{~K}$ and after a progressive increase of the external field. At low fields [Fig. 3(b)], the superconductor is in the Meissner state, and no vortices are observed inside the sample. The strong screening currents give rise to the well-known magnification of the local magnetic field close to the sample's edge. By increasing the field stepwise in intervals of $4 \mathrm{~A} / \mathrm{m}$ it is possible to determine experimentally the field $H_{\mathrm{en}}$ at which the first vortex enters the scanned area, as shown in Fig. 3(c). Because of the restricted scanning area, $H_{\mathrm{en}}$ is an overestimation of the first vortex entry field. For fields slightly above $H_{\mathrm{en}}$ the first row (i.e., the row closest to the sample's edge) of antidots is completely occupied by vortices before a vortex appears in the second row. Further increasing the external field leads to a completion of the second row of antidots. This process ends when the second row is completely occupied, beyond which a far more complex penetration is observed [Fig. 3(d)]. Indeed, (i) vortices can move further inside the sample, skipping empty rows of antidots, and (ii) double-quantized vortices appear, although they never nucleate at the sample's edge. 
(a)

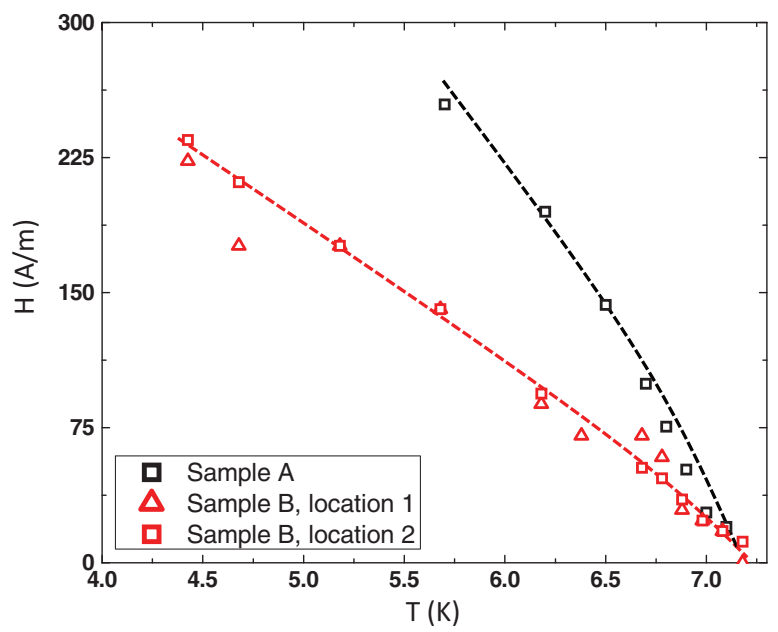

(b) $\mathrm{H}=12 \mathrm{~A} / \mathrm{m}$

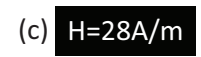

(d) $\mathrm{H}=75 \mathrm{~A} / \mathrm{m}$
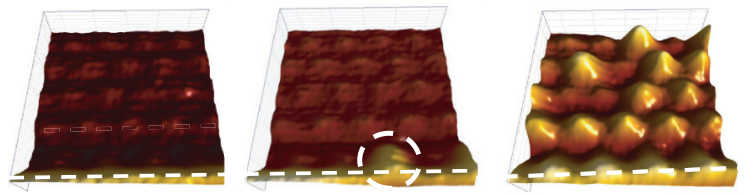

FIG. 3. (Color online) (a) Temperature dependence of the field $H_{\mathrm{en}}$ at which the first vortex is visualized in the scanning area. Dark squares are experimental data obtained for sample A, whereas triangles and light (red) squares present data obtained at two different locations for sample B. The dashed lines are a guide to the eye. (b)-(d) Scanning Hall probe microscopy images obtained at the sample's edge upon increasing external field after preparing the sample in a zero-field-cooled state at $T=7 \mathrm{~K}$. (b) In a field of $H=12 \mathrm{~A} / \mathrm{m}$ the sample is in the Meissner state, (c) at $H=28 \mathrm{~A} / \mathrm{m}$ the first vortex enters as indicated by the white circle, and (d) at $H=75 \mathrm{~A} / \mathrm{m}$ a critical state is developed. The dashed lines indicate the sample's edge.

This penetration process was associated with the terraced critical state proposed theoretically by Cooley and Grishin, ${ }^{25}$ as studied in Refs. 26 and 27. A similar process is observed in sample B. The temperature dependence of $H_{\mathrm{en}}$ for sample A (black squares) and sample B (red squares and triangles) are shown in Fig. 3(a). The different symbols for sample B reflect two distinct locations at the sample border used to obtain $H_{\mathrm{en}}$. The fact that the experimentally obtained values for $H_{\mathrm{en}}$ are independent of the measurement location demonstrates that the nucleation field is homogeneous along the sample border, validating our determination of the overall superheating field as a reliable and accurate estimation.

\section{DISCUSSION}

Now, let's compare these experimentally obtained values with the theoretical models for vortex entry. As explained above, the necessary criterium for vortex entry is the requirement that the screening-current density induced by an external magnetic field $H$ at the edge matches the GinzburgLandau depairing current density $j_{d}$ of a one-dimensional superconducting channel as given by Eq. (2). We denote with
$H_{j_{d}}$ the field at which this condition is met. We need to extend the derivation for $H_{j_{d}}$ to the case of a perpendicularly magnetized thin superconducting strip.

To determine when the current density at the edge reaches this critical value $j_{d}$ an expression for the current density at the edge has to be found. For an infinitely long, thin strip with a width $|y|<a$ and thickness $|z|<t / 2$, the complete current density distribution $J(y)$ for a perpendicularly applied external magnetic field $H \hat{z}$ was obtained as in Ref. 11 by solving numerically the Maxwell-London equations:

$$
\frac{J(y) t}{H}=\frac{y}{\sqrt{\frac{1}{4}\left[a^{2}-y^{2}\right]+\frac{\Lambda a}{\pi}}},
$$

where $\Lambda$ denotes the effective screening length of the superconducting bridge. In the thin-film limit (i.e., $\lambda \ll d$ ) the Ginzburg-Landau penetration length $\lambda$ is replaced by the effective penetration depth $\Lambda=\lambda(T)^{2} / t$. The reason for this is the reduced screening capacity of a very thin superconducting film. ${ }^{28}$ It is shown in Refs. 16, 8, and 29 that this expression derived from the Maxwell-London equations fits over a broad range of parameters with the Ginzburg-Landau solutions for the screening-current distribution as long as $H<H_{\mathrm{en}}$. Moreover, measurements of the magnetic field profile crossing the sample border when the sample is prepared in the Meissner state can be well fitted using the current distribution in Eq. (3) and Ampère's law. The agreement between the London limit approximation and the full Ginzburg-Landau solutions for the screening-current distribution in the regime $H<H_{\text {en }}$ can be augmented. First of all, our samples have a small $\kappa$ at zero temperature, and therefore, one could argue that the London limit approximation is not valid. However, due to the reduced screening efficiency in the thin-film geometry, it is appropriate to use the effective penetration depth $\Lambda$. The increase of $\Lambda$ with increasing temperatures is stronger than the increase of $\xi$. As a result, $\kappa_{\text {eff }}=\Lambda / \xi$ exceeds $\kappa(0)$ at the experimental temperatures. Second, the condition $H<H_{\mathrm{en}}$ means that there is a limited kinetic reduction of the density of states at the edge due to current-induced depairing. Moreover, the periodic lattice of antidots forces the pair density to be nearly constant in the space between antidots due to the boundary conditions at the superconductor-insulator interface. ${ }^{1}$ A more detailed discussion on the London approximation for the first vortex penetration in a thin-film geometry, derived from first principles, will be presented elsewhere. ${ }^{30}$

However, we are interested in the condition that the induced screening current at the edge of the sample approaches $j_{d}$. It is expected then that Eq. (3) fails to describe the correct relation between the induced screening-current density and the applied magnetic field, particularly in the neighborhood of the edge, as current-induced depairing will locally alter the density of states dramatically. However, at distances larger than the effective penetration depth away from the sample edge, the current density decreases quickly below the critical value for depairing, and the London limit is restored. Since the global response of the superconducting strip to an applied magnetic field is determined by the whole current distribution, it is expected that in the limit $2 a \gg \Lambda$, the London limit gives a very good description of the demagnetizing effects as it is valid for the major part of the sample's volume. However, 


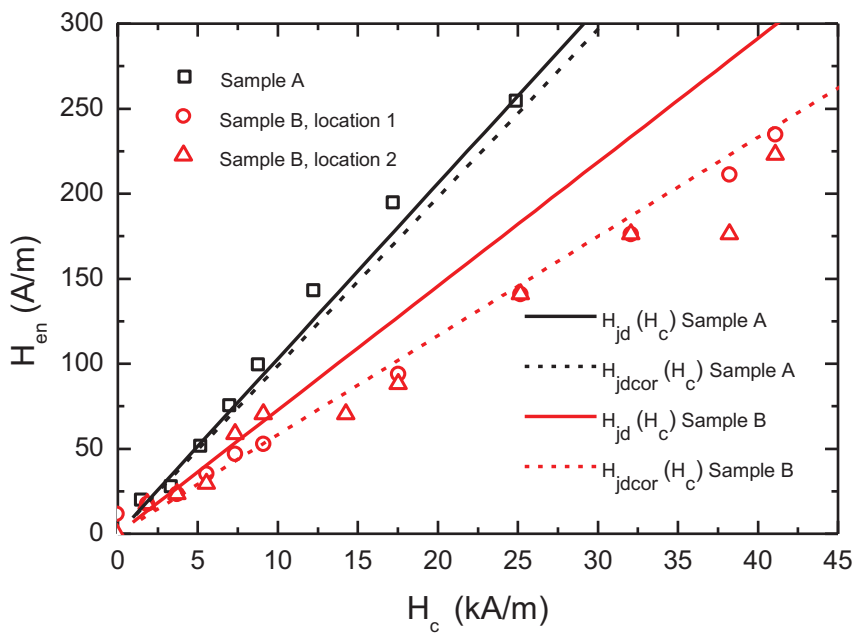

FIG. 4. (Color online) The obtained experimental data for the first vortex entry field versus $H_{c}(T)$ obtained by Decker et al. ${ }^{31}$ is shown for sample A (squares) and for two locations at the border for sample B (circles and triangles). The theoretical curves obtained from Eq. (5) for samples A and B with $C=\frac{3 \sqrt{6}}{4}$ are shown by the solid black and red lines, respectively. The dashed black and red lines take into account the effect of the pattern-induced enhancement of the screening-current density for samples A and B, respectively.

the condition for vortex entry is a local one, and we have to take into account the effect of current-induced depairing at the edge. In the first approximation, we will introduce this effect by using a new effective Ginzburg-Landau penetration length $\lambda^{\prime}=C \lambda$ as a cutoff factor at the edge. Here, $C$ is a constant bigger than unity since due to current-induced depairing the screening currents are weakened and the magnetic field is less effectively screened. Therefore, using Eq. (3), the current at the edge $(y=a)$ of the sample is given by

$$
j_{\text {edge }}=\left[\frac{1}{C \lambda(T)}\right]\left[\sqrt{\frac{a \pi}{t}}\right] H .
$$

As such, rewriting $j_{\text {edge }}$ and $H$ as $J_{d}$ and $H_{j d}$ and using Eq. (2), we obtain an expression for $H_{j d}$ :

$$
H_{j_{d}}=\left[\frac{C}{\frac{3 \sqrt{6}}{4}}\right]\left[\sqrt{\frac{t}{a \pi}}\right] H_{c}(T) .
$$

As a result $H_{j_{d}}$ is determined by the temperature dependence of $H_{c}(T)$, a geometric demagnetizing factor, and a constant related to the depletion of the condensate of our superconducting film.

The temperature dependence of $H_{c}$ for bulk $\mathrm{Pb}$ samples was measured by Decker et al., leading to an extrapolated value of $H_{c}(0)=63.87 \mathrm{kA} / \mathrm{m}$ at $T=0 \mathrm{~K} .{ }^{31}$ This value is, within the experimental error, identical to $H_{c}(0)=64.06 \mathrm{kA} / \mathrm{m}$ given in Ref. 32 for an amorphous $\mathrm{Pb}$ film. In Fig. 4, the experimentally obtained values for $H_{\mathrm{en}}(T)$ are plotted versus $H_{c}(T)$ obtained by Decker et al. ${ }^{31}$ According to Eq. (5) and a linear fit of the data $H_{\mathrm{en}}(T)$ for sample A (squares) versus $H_{c}(T)$, a slope of $(10.6 \pm 0.4) \times 10^{-3}$ is obtained. Taking into account the width $2 a=300 \mu \mathrm{m}$ and thickness $t=50 \mathrm{~nm}$ of the sample, we obtain $C=1.03 \times \frac{3 \sqrt{6}}{4}$.
This means that if we enhance the Ginzburg-Landau penetration depth by this factor $C$ to take into account the effect of current-induced pairing within the London model expression for the screening-current density at the edge, a nice match is found between the experimental results $H_{\mathrm{en}}(T)$ and the theoretically estimated superheating field $H_{j_{d}}(T)$, as indicated by the solid black line in Fig. 4. The enhancement factor of the effective penetration depth $C$ is in a good agreement with the factor appearing in the Ginzburg-Landau expression for $J_{d}$ due to current-induced depairing [Eq. (2)]. This is not a surprise since in a bulk sample, in the first approximation the field penetration at the edge can be assumed as an exponential decrease of the magnetic field, $B=B_{0} e^{-y / \lambda_{\text {edge }}}$, where $\lambda_{\text {edge }}$ is the locally increased penetration depth compared to the bulk value due to current induced depairing. In the case of a thick superconductor, using an exponential penetration of magnetic field, the physical meaning of $\lambda_{\text {edge }}$ is nicely shown by applying Ampère's law:

$$
|\nabla \times B|=\frac{B_{0}}{\lambda_{\text {edge }}}=\mu_{0} J_{\text {edge }} .
$$

As the current density at the edge can be at most the GinzburgLandau depairing current density, which is lower than the London depairing current density by a factor of $(3 \sqrt{6} / 4)$, the field will be screened over a larger distance. It is clear from Eqs. (6) and (2) that the new length scale on which the field varies at the edge is given by

$$
\lambda_{\text {edge }}=(3 \sqrt{6} / 4) \lambda(T) .
$$

In order to corroborate these results we performed similar measurements on sample B. The obtained experimental data for the first vortex entry field versus $H_{c}(T)$ obtained by Decker et al. $^{31}$ is plotted in Fig. 4 (triangles and circles). As expected, a clear reduction of $H_{\mathrm{en}}$ is observed [see also Fig. 3(a)], related to the larger width of sample $B$ (i.e., increased demagnetization field). However, the value we obtain for $C=0.74 \times \frac{3 \sqrt{6}}{4}$ from a linear fit of the experimental data obtained for the first vortex entry field in sample B deviates significantly from the value we obtained for $C$ in sample A. Ascribing this factor $C$ to the current-induced depairing effect alone, one does not expect any difference in this factor for both samples.

Due to a different antidot array geometry and effective antidot width, a more pronounced effect is expected in sample B compared to sample A. The periodicity of antidots perpendicular to current circulation is 5 and $3 \mu \mathrm{m}$ for sample A (triangular array) and sample B (square array), respectively. For sample A the enhancement factor of the edge screeningcurrent density is $5 \mu \mathrm{m} / 4.77 \mu \mathrm{m}=1.05$, while for sample B this factor is $3 \mu \mathrm{m} / 2.4 \mu \mathrm{m}=1.25$. In Fig. 4 the dashed curves incorporate this effect for both samples, while in the solid lines this effect is neglected. It is clear that by taking this effect into account, a nice fit is recovered. The importance of these local enhancements of the current density at the edge due to the presence of the antidots is evidenced by observed modulations of the edge field in the vortex-free state.

\section{CONCLUSION}

In conclusion, we have visualized the first penetration of vortices in superconducting films with antidots by scanning 
Hall probe microscopy. The studied thin-film geometry and the presence of antidots have allowed us to argue, using the London approximation, that the first vortex penetration occurs when the condition $j_{\text {edge }}=j_{d}$ is met.

From the obtained results, we can propose the following picture for the observed vortex entry process. At low values of the applied field, the superconductor is in the Meissner state, where supercurrents circulate in the whole sample with a decreasing density toward the center. When the applied field reaches $H_{j_{d}}$ and the edge current density approaches its depairing value (more accurately, it should be the current density averaged over a $\xi$ distance), there is a local breakdown of superconductivity and a first vortex nucleation at the edge near an antidot, where the Meissner current density takes a local maximum due to the reduced current path width. The nucleated vortex is immediately depinned from the edge and is caught by the nearest antidot, where it is observed. Since the vortex current has its sense opposite to the Meissner current, the involved edge current density decreases back to below its depairing value, and the nucleation of the second vortex occurs in another place and so on.

\section{ACKNOWLEDGMENTS}

We thank John R. Clem and D.-X. Chen, C. Navau, N. Del-Valle, and A. Sanchez from the Departament de Fisica, Universitat Autonoma de Barcelona, for fruitful discussions. We acknowledge the Methusalem funding by the Flemish government and the Flemish Science Foundation for financial support. We also acknowledge the support from the MP1201 COST action. J.G. and J.V.d.V. acknowledge support from FWO-Vl. A.V.S. and R.B.G.K. acknowledge the support of Wallonie-Bruxelles International, FNRS, and the French Ministry of Foreign Affairs within the framework of the bilateral project Hubert Curien. The work of A.V.S. was partially supported by "Crédit de démarrage" of the ULg.
${ }^{*}$ These authors equally contributed to this work.
${ }^{1}$ P. G. de Gennes, Superconductivity of Metals and Alloys (Addison-
Wesley, Reading, MA, 1989).
${ }^{2}$ C. P. Bean and J. D. Livingston, Phys. Rev. Lett. 12, 14 (1964).
${ }^{3}$ H. J. Fink and A. G. Presson, Phys. Rev. 182, 498 (1969).
${ }^{4}$ F. P. Lin and A. Gurevich, Phys. Rev. B 85, 054513 (2012).
${ }^{5}$ L. Kramer, Phys. Rev. 170, 475 (1968).
${ }^{6}$ P. G. de Gennes, Solid State Commun. 3, 127 (1965).
${ }^{7}$ L. G. Aslamazov and S. V. Lemnitskii, Zh. Eksp. Teor. Fiz. 84, 2216
(1983) [JETP 57, 1291 (1983)].

${ }^{8}$ D. Y. Vodolazov, I. L. Maksimov, and E. H. Brandt, Europhys. Lett. 48, 313 (1999).

${ }^{9}$ E. Zeldov, A. I. Larkin, V. B. Geshkenbein, M. Konczykowski, D. Majer, B. Khaykovich, V. M. Vinokur, and H. Shtrikman, Phys. Rev. Lett. 73, 1428 (1994).

${ }^{10}$ M. Benkraouda and J. R. Clem, Phys. Rev. B 53, 5716 (1996).

${ }^{11}$ B. L. T. Plourde, D. J. Van Harlingen, D. Yu. Vodolazov, R. Besseling, M. B. S. Hesselberth, and P. H. Kes, Phys. Rev. B 64, 014503 (2001).

${ }^{12}$ A. S. Joseph and W. J. Tomasch, Phys. Rev. Lett. 12, 219 (1964).

${ }^{13}$ R. W. de Blois and W. de Sorbo, Phys. Rev. Lett. 12, 499 (1964).

${ }^{14}$ A. S. Joseph, W. J. Tomasch, and H. J. Fink, Phys. Rev. 157, 315 (1967).

${ }^{15}$ D. Cerbu, V. N. Gladilin, J. Cuppens, J. Tempere, J. T. Devreese, V. V. Moshchalkov, A. V. Silhanek, and J. Van de Vondel, New J. Phys. 15, 063022 (2013).

${ }^{16}$ D. Y. Vodolazov, I. L. Maksimov, and E. H. Brandt, Phys. C 384, 211 (2003).

${ }^{17}$ M. Benkraouda and J. R. Clem, Phys. Rev. B 58, 15103 (1998).

${ }^{18}$ S. Raedts, A. V. Silhanek, M. J. Van Bael, and V. V. Moshchalkov, Phys. Rev. B 70, 024509 (2004); S. Raedts, A. V. Silhanek, V. V. Moshchalkov, J. Moonens, and L. H. A. Leunissen, ibid. 73, 174514 (2006).
${ }^{19}$ J. I. Vestgården, D. V. Shantsev, Y. M. Galperin, and T. H. Johansen, Phys. Rev. B 77, 014521 (2008); K. A. Lőrincz, M. S. Welling, J. H. Rector, and R. J. Wijngaardenl, Phys. C 411, 1 (2004).

${ }^{20}$ C. Reichhardt, C. J. Olson, and F. Nori, Phys. Rev. B 57, 7937 (1998).

${ }^{21}$ C. Reichhardt and N. Gronbech-Jensen, Phys. Rev. B 63, 054510 (2001).

${ }^{22}$ A. N. Grigorenko, G. D. Howells, S. J. Bending, J. Bekaert, M. J. Van Bael, L. Van Look, V. V. Moshchalkov, Y. Bruynseraede, G. Borghs, I. I. Kaya, and R. A. Stradling, Phys. Rev. B 63, 052504 (2001).

${ }^{23}$ S. B. Field, S. S. James, J. Barentine, V. Metlushko, G. Crabtree, H. Shtrikman, B. Ilic, and S. R. J. Brueck, Phys. Rev. Lett. 88, 067003 (2002)

${ }^{24}$ A. N. Grigorenko, S. J. Bending, M. J. Van Bael, M. Lange, V. V. Moshchalkov, H. Fangohr, and P. A. J. de Groot, Phys. Rev. Lett. 90, 237001 (2003).

${ }^{25}$ L. D. Cooley and A. M. Grishin, Phys. Rev. Lett. 74, 2788 (1995).

${ }^{26}$ V. V. Moshchalkov, M. Baert, V. V. Metlushko, E. Rosseel, M. J. Van Bael, K. Temst, Y. Bruynseraede, and R. Jonckheere, Phys. Rev. B 57, 3615 (1998).

${ }^{27}$ A. V. Silhanek, J. Gutierrez, R. B. G. Kramer, G. W. Ataklti, J. Van de Vondel, and V. V. Moshchalkov, and A. Sanchez, Phys. Rev. B 83, 024509 (2011).

${ }^{28}$ J. Pearl, Appl. Phys. Lett. 5, 65 (1964).

${ }^{29}$ D. Y. Vodolazov, Tech. Phys. Lett. 25, 838 (1999).

${ }^{30}$ D.-X. Chen, C. Navau, N. Del-Valle, and A. Sanchez (to be published).

${ }^{31}$ D. L. Decker, D. E. Mapother, and R. W. Shaw, Phys. Rev. 112, 1888 (1958).

${ }^{32}$ M. Tinkham, Phys. Rev. 129, 2413 (1963). 